\title{
Compton scattering x-ray sources driven by laser wakefield acceleration
}

\author{
F. V. Hartemann, D. J. Gibson, and W. J. Brown* \\ Lawrence Livermore National Laboratory, Livermore, California 94550, USA \\ A. Rousse, K. Ta Phuoc, V. Mallka, and J. Faure \\ Ecole Nationale Supérieure des Techniques Avancées, Laboratoire d'Optique Appliquée, 91761 Palaiseau, France
}

\section{A. Pukhov}

Institute for Theoretische Physik I, University of Dusseldorf, 40225 Dusseldorf, Germany

(Received 19 October 2005; revised manuscript received 15 December 2006; published 24 January 2007)

\begin{abstract}
Recent laser wakefield acceleration experiments have demonstrated the generation of femtosecond, nano-Coulomb, low emittance, nearly monokinetic relativistic electron bunches of sufficient quality to produce bright, tunable, ultrafast x-rays via Compton scattering. Design parameters for a proof-of-concept experiment are presented using a three-dimensional Compton scattering code and a laser-plasma interaction particle-in-cell code modeling the wakefield acceleration process; x-ray fluxes exceeding $10^{21} \mathrm{~s}^{-1}$ are predicted, with a peak brightness $>10^{19}$ photons $/\left(\mathrm{mm}^{2} \mathrm{mrad}^{2} \mathrm{~s} 0.1 \%\right.$ bandwidth $)$ ).
\end{abstract}

\section{INTRODUCTION}

The generation of bright, ultrafast, tunable, hard x-rays from truly tabletop systems is a research goal of considerable interest, currently pursued by a number of groups worldwide. Such novel x-ray sources would have a wide variety of applications, ranging from dynamic diffraction studies of laser-pumped materials on the femtosecond time scale to time-resolved molecular dynamics [1-4] and advanced biomedical imaging [5].

Among different proposed schemes to generate ultrafast $\mathrm{x}$-rays, such as laser-driven $\mathrm{K}_{\alpha}$ sources [2,6-8], highharmonic generation [9], or free-electron lasers [10], Compton scattering possesses a unique set of valuable characteristics, including compactness, wide tunability, femtosecond time scale, and the potential for high brightness. This method has already been demonstrated using picosecond and femtosecond lasers synchronized to an rf linear accelerator (linac) [11-15]. Intense picosecond x-ray beams can be obtained in that case using head-on interaction geometries. The duration of the x-ray pulses depends on size of the interaction area, which fully depends on the size of the laser and particle beams as well as the angle of interaction; in particular, for head-on collisions $\left(180^{\circ}\right.$ interaction), the $\mathrm{x}$-ray pulse duration is essentially equal to the electron bunch length. Different crossing techniques can then be used to keep the electron-photon superposition area small enough and produce subpicosecond x-ray pulses [16]; however, the x-ray flux becomes strongly diminished if the entire electron beam does not overlap with the laser pulse to produce the Compton scattering radiation.

\footnotetext{
*Current address: Lincoln Laboratory, MIT, Lexington, MA 02420 .
}

Recently, three different groups [17-19] have demonstrated the successful production of high charge $(1 \mathrm{nC})$, high energy ( $>50 \mathrm{MeV})$, nearly monokinetic $(\Delta \gamma / \gamma \leq$ $10 \%)$ laser-produced plasma electron bunches using terawatt-class, few Joules, 30 fs chirped-pulse amplification (CPA) laser systems. These electron beams have a low divergence angle (few mrad), and the source size is very small (few $\mu \mathrm{m}$ ); thus, the normalized emittance is comparable to, or better than, that obtained in conventional highbrightness linacs. Furthermore, the electron pulse duration is extremely short, of the order of a few tens of femtoseconds; this unique set of characteristics opens the path toward practical, compact, high-brightness hard x-ray sources such as the all-optical Compton scattering scheme discussed in this paper, and first proposed by Esarey et al. [20].

The design parameters of a proof-of-principle experiment are presented, whereby hard x-rays are generated by colliding ultrashort electron bunches obtained via laser wakefield acceleration (LWFA) [17-19,21-27] with laser pulses produced by a TW-class CPA laser, as shown schematically in Fig. 1. The advantages of using laser acceleration to produce the relativistic electrons are manifold: first, the $\sim 1 \mathrm{nC}$ electron bunches produced in this manner are extremely short, thus leading to intense femtosecond x-ray flashes by using the entire electron bunch charge; second, the electrons can be synchronized with the Compton scattering drive laser with great accuracy; third, LWFA is a high-gradient process, allowing for a very compact system; finally, sufficiently low emittance and monokinetic beams can produce bright, broadband x-ray pulses that could be used for a number of interesting applications, including femtosecond Laue diffraction. 


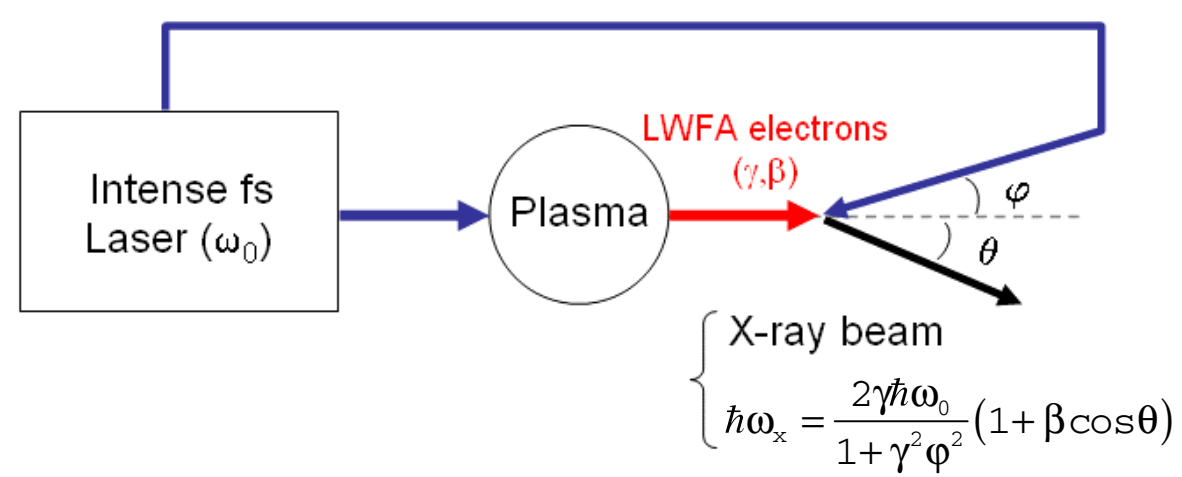

FIG. 1. (Color) Schematic of a Compton scattering x-ray experiment using LWFA electrons. The energy of the x-rays, $\hbar \omega_{x}$, depends on the angle between the laser and electron beams, $\varphi$, as well as on the angle of observation from the electron beam direction, $\theta$, and the electron energy, $\gamma m_{0} c^{2}$.

This work is further motivated by recent Thomson scattering experiments using a laser-accelerated electron beam, where photons scattered by $\mathrm{MeV}$ electrons were measured in the $0.4-2 \mathrm{keV}$ range [28].

In Compton scattering, the basic interaction relies on the double Doppler upshift of incident photons by relativistic electrons: for head-on collisions, the energy of the scattered $\mathrm{x}$-rays is given by $\hbar \omega_{x} \simeq 4 \gamma^{2} \hbar \omega_{0}$, where $\gamma=1+$ $\mathrm{eV} / \mathrm{m}_{0} \mathrm{c}^{2}$ is the relativistic factor of the electrons accelerated to a potential $V$, and $\omega_{0}$ is the frequency of the incident light. For example, for $800 \mathrm{~nm}$ light produced by a $\mathrm{Ti}: \mathrm{Al}_{2} \mathrm{O}_{3}$ laser, the incident photon energy is $1.55 \mathrm{eV}$; a $50 \mathrm{MeV}$ electron beam will generate $60 \mathrm{keV}$ $\mathrm{x}$-rays for head-on collisions. In addition, for the same interaction geometry, the x-ray pulse duration is essentially equal to the electron bunch length, thus offering the potential to generate femtosecond $\mathrm{x}$-ray flashes; this particular interaction geometry offers other advantages over crossed beam setups, including better overlap and higher $\mathrm{x}$-ray photon number, as well as improved performance against timing jitter. Because the interaction is based on the relativistic Doppler effect, however, the electron beam quality plays an important role in the brightness of the $\mathrm{x}$-rays.

In this paper, the properties of the electron beam used for the Compton scattering calculations are derived from three-dimensional (3D) particle-in-cell (PIC) simulations $[29,30]$ that fit the experimental data of the more recent LWFA experiments [17]. The brightness scaling of Compton scattering and other salient theoretical features are then briefly outlined, and the 3D time and frequency domain Compton scattering code structure is summarized; this code has been extensively benchmarked against recent Compton scattering experiments performed at Lawrence Livermore National Laboratory (LLNL) using an S-band linac operating in the $60-80 \mathrm{MeV}$ range, a high-brightness S-band photoinjector, and a terawatt-class, $810 \mathrm{~nm}$ Ti:sapphire laser [31-34]. Finally, the two codes are interfaced to predict the properties of the x-ray pulses that a Compton scattering source driven by laser wakefield ac- celeration will produce experimentally, and conclusions are drawn.

\section{ELECTRON BEAM PARAMETERS}

Recent experiments have shown that by focusing an ultraintense and ultrashort laser pulse into an underdense plasma, quasimonoenergetic electron bunches can be generated. In the experiment described in Ref. [17], the laser pulse had a duration of $33 \pm 2 \mathrm{fs}$ (FWHM), and contained $1 \mathrm{~J}$ of laser energy at a central wavelength of $820 \mathrm{~nm}$. It was focused onto the edge of a 3-mm-long supersonic helium gas jet using an $f / 18$ off-axis parabola. The diffractionlimited focal spot had a diameter of $21 \mu \mathrm{m}$ FWHM, producing a vacuum-focused laser intensity of $3.2 \times$ $10^{18} \mathrm{~W} / \mathrm{cm}^{2}$. For a plasma density of $6 \times 10^{18} \mathrm{~cm}^{-3}$, an electron bunch with a peak energy of $170 \mathrm{MeV}$ was produced, with an energy spread measured at 24\% FWHM, limited by the electron spectrometer resolution. Experimentally, this regime could be reached in a narrow range of parameters: stretching the pulse duration above $50 \mathrm{fs}$ was sufficient to lose the peaked energy distribution. Similarly, when the electron density was increased from $6 \times 10^{18} \mathrm{~cm}^{-3}$ to $7.5 \times 10^{18} \mathrm{~cm}^{-3}$, the energy distribution became a broad plateau. This suggests that the monoenergetic electron beam could be obtained preferentially when the laser pulse length was as short as possible, and when the laser intensity was highest.

Three-dimensional (3D) PIC simulations using the code "Virtual Laser Plasma Laboratory" [29,30] were used to closely match the data of the experiments [17]; typical simulation results are shown in Figs. 2 and 3, which illustrate the main features of the electron beam phase space produced in the so-called LWFA "cavitation" regime. A summary of the experimental and numerical parameters for the LWFA accelerator is shown in Table I. The narrow energy spread electron beam results from the highly nonlinear interaction of the laser with the plasma. As the pulse propagates in the plateau region of a gas jet, it self-focuses and undergoes longitudinal compression by 

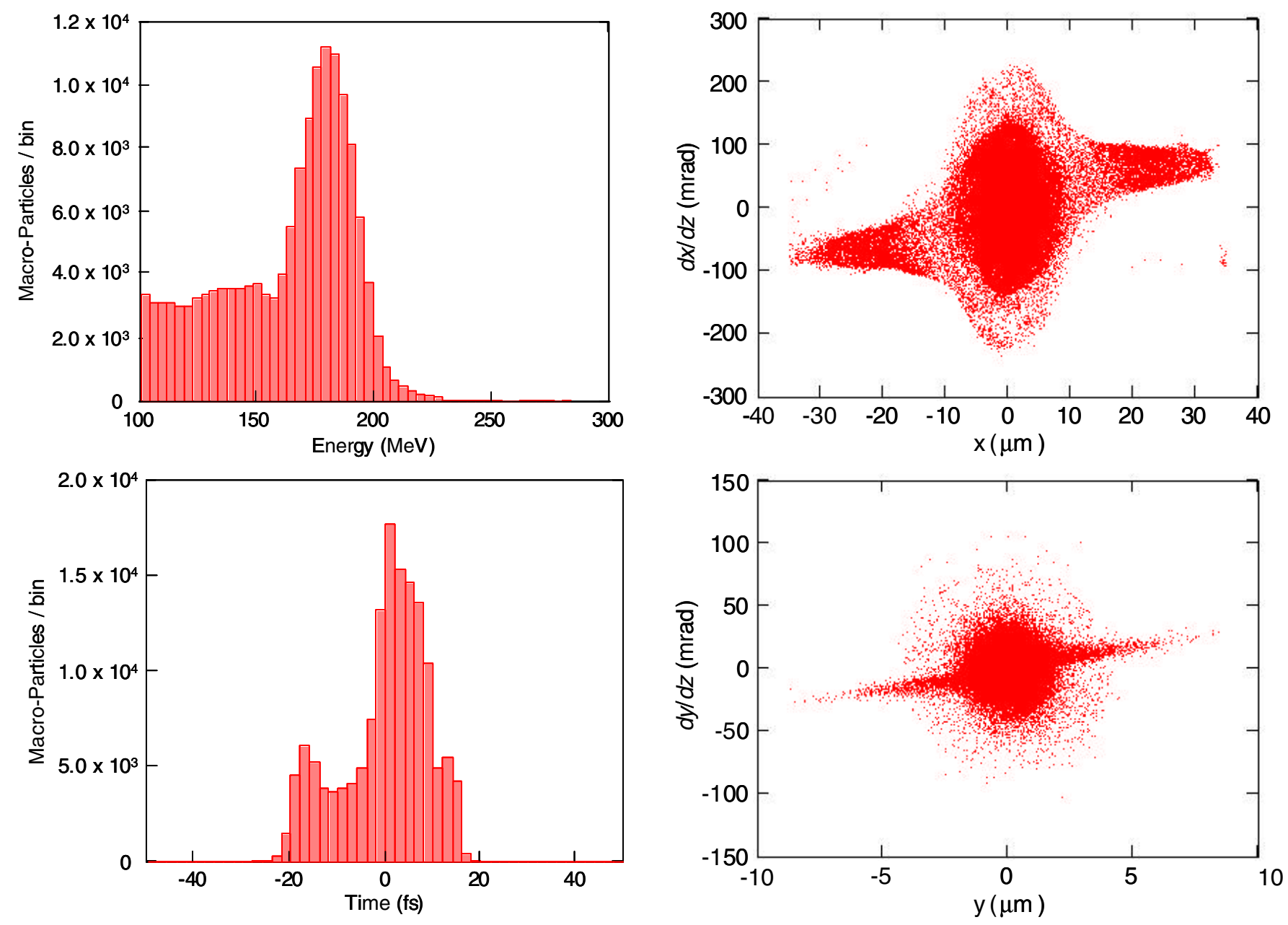

FIG. 2. (Color) LWFA phase space, as simulated by 3D PIC code. Top: electron beam energy spectrum. Bottom: temporal electron pulse profile.

plasma waves. This decreases the effective radius of the laser pulse and increases the laser intensity by 1 order of magnitude. This compressed laser pulse is now resonant with the plasma wave and it drives a highly nonlinear wakefield: the laser ponderomotive potential expels the plasma electrons radially and leaves a cavitated region behind (this is referred to as the "cavitation" or "blowout" regime). In this regime, the 3D structure of the wakefield resembles a plasma bubble. As the electron density at the walls of the bubble becomes large, wave breaking occurs and electrons are injected and accelerated inside the bubble. As the number of trapped electrons increases, the bubble elongates. Its effective group velocity decreases, and electrons start to dephase with respect to the accelerating field. This dephasing causes electron selfbunching in phase space, which results in a nearly monokinetic peak in the energy spectrum $[25-27,29,30]$.

In a very recent experiment, we have demonstrated that the injection and subsequent acceleration of electrons can be controlled by using a second and counterpropagating

FIG. 3. (Color) LWFA transverse electron beam phase space, as simulated by 3D PIC code.

laser pulse [35]. The collision of the two laser pulses provides a preacceleration stage which provokes the injection of electrons into the plasma wakefield [36]. The experimental results show that the electron beams obtained in this manner are collimated (5 mrad divergence), monoenergetic (with energy spread $<10 \%$ ), tunable (between 15 and $250 \mathrm{MeV}$ ) and, most importantly, stable (with standard deviation lower than $10 \%$ for all parameters). In addition, simulations and experimental results strongly suggest that these electron bunches are shorter than $10 \mathrm{fs}$. Such high quality, tunable, and stable electron beams would be of great interest for the generation of Compton scattering ultrafast X-rays.

The LWFA electron bunch transverse dimensions are inferred experimentally from the laser spot size. The emittance is difficult to measure experimentally because it is hard to directly measure the size of the electron source. In PIC simulations, the divergence is typically $5 \mathrm{mrad}$ (like in the experiments) and the source size is $2-3$ microns. The emittance is then $\varepsilon=2 \mu \mathrm{m} \times 5 \mathrm{mrad}=10^{-2} \mathrm{~mm} \mathrm{mrad}$, and the normalized emittance for a $200 \mathrm{MeV}$ beam 
TABLE I. LWFA parameters from Ref. [17].

\begin{tabular}{lll}
\hline \hline \multicolumn{1}{c}{ Physical parameters } & \multicolumn{1}{c}{ Experiment } & \multicolumn{1}{c}{ PIC simulation } \\
\hline Drive laser pulse wavelength & $820 \mathrm{~nm}$ & $800 \mathrm{~nm}$ \\
Drive laser pulse energy & $1 \mathrm{~J}$ & $1 \mathrm{~J}$ \\
Drive laser pulse duration & $33 \pm 2 \mathrm{fs}(\mathrm{FWHM})$ & $21 \mathrm{fs}$ \\
Plasma density & $6.0 \times 10^{18} \mathrm{~cm}^{-3}$ & $6.0 \times 10^{18} \mathrm{~cm}^{-3}$ \\
Electron bunch charge & $0.5 \pm 0.2 \mathrm{nC}$ & $0.5 \mathrm{nC}$ \\
Electron bunch duration & Not measured & $12 \mathrm{fs}$ \\
Electron bunch energy & $170 \mathrm{MeV}$ & $170 \mathrm{MeV}$ \\
Relative energy spread & $24 \%(\mathrm{FWHM})$ & $15 \%(\mathrm{FWHM})$ \\
Electron bunch divergence & $6-10 \mathrm{mrad}(\mathrm{FWHM})$ & $5-10 \mathrm{mrad}(\mathrm{FWHM})$ \\
Electron bunch radius & $2-3 \mu \mathrm{m}(\mathrm{FWHM}$, inferred) & $2-3 \mu \mathrm{m}(\mathrm{FWHM})$ \\
\hline \hline
\end{tabular}

$(\gamma=400)$ is $\varepsilon_{n}=\gamma \times \varepsilon=4 \mathrm{~mm}$ mrad, which is similar to the emittance of $\mathrm{rf}$ guns. The LWFA emittance was actually measured, under slightly different conditions, in Ref. [37]; we found a normalized emittance of a few $\mathrm{mm}$ mrad at $50 \mathrm{MeV}$, which confirms the expected values.

\section{COMPTON SCATTERING THEORY AND NUMERICAL CODE}

In Compton scattering, the Doppler upshifted frequency of the scattered radiation can be readily determined by considering energy-momentum conservation: $m_{0} c u_{\mu}^{0}+$ $\hbar k_{\mu}^{0}=m_{0} c u_{\mu}^{s}+\hbar k_{\mu}^{s}$, where $u_{\mu}^{0}$ is the electron 4-velocity before the interaction, $k_{\mu}^{0}$ is the 4-wave-number of the incident photon, and where $u_{\mu}^{s}$ and $k_{\mu}^{s}$ are the corresponding quantities after the scattering event; one finds that $k_{\mu}^{s}\left(u_{\mu}^{0}+\lambda_{C} k_{\mu}^{0}\right)=k_{\mu}^{0} u_{0}^{\mu}$. In the case where recoil is negligible, we have $\lambda_{C} k_{0} \ll 1$, and one recovers the wellknown Doppler effect:

$$
\frac{k_{s}}{k_{0}}=\frac{\gamma_{0}-u_{0} \cos \varphi}{\gamma_{0}-u_{0} \cos \theta}
$$

where $\varphi$ and $\theta$ are the incidence and scattering angles, respectively.

The local number of $x$-ray photons scattered per unit time and volume is given by the product of the electron beam 4-current, $j_{\mu}\left(x_{\nu}\right)=e c n_{e}\left(x_{\nu}\right) u_{\mu} / \gamma$, and the incident photon 4-flux, $\Phi_{\mu}\left(x_{\nu}\right)=c n_{\lambda}\left(x_{\nu}\right) k_{\mu} / \omega$ :

$$
\frac{d^{4} N_{x}\left(x_{\nu}\right)}{d^{4} x_{\nu}}=\frac{\sigma}{e c} j_{\mu}\left(x_{\nu}\right) \Phi^{\mu}\left(x_{\nu}\right)=\frac{\sigma c}{\gamma \omega} n_{e}\left(x_{\nu}\right) n_{\lambda}\left(x_{\nu}\right) u_{\mu} k^{\mu} .
$$

Here, $u_{\mu}=d x_{\mu} / c d \tau=(\gamma, \mathbf{u})$ is the electron 4-velocity, $k_{\mu}=(\omega / c, \mathbf{k})$ is the incident photon 4-wave-number, and $\sigma=8 \pi r_{0}^{2} / 3$ is the Compton scattering cross section.
The local spectral brightness is

$$
\begin{aligned}
\frac{d^{6} N_{x}}{d^{4} x_{\mu} d \Omega_{x} d \omega_{x}}= & \frac{d \sigma}{d \Omega_{x}}\left(1-\beta \frac{c \mathbf{k}}{\omega}\right) n_{\lambda}\left(x_{\mu}\right) n_{e}\left(x_{\mu}\right) \\
& \times \delta\left(\omega_{x}-\omega \frac{\kappa}{\kappa_{x}}\right),
\end{aligned}
$$

where $\kappa=\gamma-\mathbf{u} \cdot \frac{c \mathbf{k}}{\omega}$ and $\kappa_{x}=\gamma-\mathbf{u} \cdot \hat{\mathbf{n}}_{x}$ are the electron light-cone variables [38] with respect to the incident and scattered photons, respectively.

For Gaussian beams propagating paraxially, the peak brightness can be derived analytically under the assumption that the energy spread and emittance remain small:

$$
\begin{aligned}
\hat{B}_{x}= & \frac{4 \times 10^{-15}}{\pi^{2}} \frac{\gamma_{0}^{2}}{\varepsilon^{2}} \frac{N_{e} N_{\lambda}}{\Delta \tau} \frac{r_{0}^{2}}{w_{0}^{2}} \\
& \times \exp \left\{\frac{\chi-1}{2 \chi \Delta u_{\perp}^{2}}\left[2+\frac{\delta \omega^{2}+\delta \gamma^{2} \chi^{2}}{2 \chi(\chi-1) \Delta u_{\perp}^{2}}\right]\right\} \\
& \times\left[1-\Phi\left\{\frac{\chi-1}{\sqrt{\delta \omega^{2}+\delta \gamma^{2} \chi^{2}}}\left[1+\frac{\delta \omega^{2}+\delta \gamma^{2} \chi^{2}}{2 \chi(\chi-1) \Delta u_{\perp}^{2}}\right]\right\}\right] \\
& \times \frac{\eta e^{1 / \eta^{2}}\left[\Phi\left(\frac{1}{\eta}\right)-1\right]-\mu e^{1 / \eta^{2}}\left[\Phi\left(\frac{1}{\eta}\right)-1\right]}{\mu^{2}-\eta^{2}} .
\end{aligned}
$$

Here, $N_{e}=q / e$ is the number of electrons in the bunch, $\Delta \tau$ is the $1 / e^{2}$ bunch duration, $\sigma_{r}$ is the $1 / e^{2}$ radius at focus, and the inverse beta function is given in terms of the normalized emittance, $\varepsilon$, beam focal radius, and energy, $\gamma_{0}$, by $k_{f}=\beta_{f}^{-1}=\varepsilon / \gamma_{0} \sigma_{r}^{2} ; N_{\lambda}=W / \hbar \omega_{0}$ is the total number of photons in the laser pulse, $\Delta t$ is the $1 / e^{2}$ pulse duration, and is related to the bandwidth as $\Delta t \Delta \omega=\sqrt{2}$, in the case of a Fourier transform-limited pulse, $w_{0}$ is the $1 / e^{2}$ focal radius, and $z_{0}=\pi w_{0}^{2} / \lambda_{0}$ is the associated Rayleigh length; $\chi=\omega_{x} / 4 \gamma_{0}^{2} \omega_{0}$ is the normalized Doppler-upshifted frequency, $\delta \omega=\Delta \omega / \omega_{0}$ is the relative spectral width of the drive laser pulse, and $\delta \gamma=2 \Delta \gamma / \gamma_{0}$ is the scaled relative energy spread; $\Delta u_{\perp} \sigma_{r}=\varepsilon u_{0} / \gamma_{0} \simeq$ $\varepsilon$; finally, we have also defined the normalized inverse beta 
function, $\eta=k_{f} c \Delta t / 2 \sqrt{2}$, and the normalized inverse Rayleigh length, $\mu=c \Delta t / 2 \sqrt{2} z_{0}$.

This result clearly underscores the importance of the electron beam quality: the $\mathrm{x}$-ray brightness is proportional to that of the electron bunch. In particular, the x-ray brightness scales inversely quadratically with the physical emittance, $\varepsilon / \gamma_{0}$, and linearly with the electron bunch focused current density. Therefore, the ultrashort, high charge, low emittance electron beams produced by laser wakefield acceleration are good candidates to drive compact Compton scattering $\mathrm{x}$-ray sources, provided their energy spread is sufficiently low; for a more detailed discussion and a comparison with conventional rf linacs, we refer the reader to Sec. IV. The details of the derivations leading to the results given above can be found in Ref. [31].

An extensive description of the three-dimensional time and frequency domain x-ray code used here to model the main characteristics of the radiation produced by colliding a relativistic electron bunch produced by LWFA with an intense laser pulse is given in Ref. [32]; in the present paper, a brief overview is given for completeness.

For realistic electron and laser beams, Eq. (3) must be integrated over the entire phase space of each beam to yield the corresponding x-ray brightness, within the context of an incoherent superposition.

In its most general form, the program calculates the number of photons scattered into a given solid angle and within a range of scattered frequencies at each time step by summing over all incident wave vectors within the laser pulse, which accurately models the laser pulse phase space. This is performed for all the electrons in the bunch, which are represented by a series of macroparticles with a chargeto-mass ratio equal to $e / m_{0}$. Furthermore, the zeroth-order (no laser) electron motion through the laser focus is assumed to be ballistic, which is a good assumption given the fact that the relativistic plasma wavelength of the electron bunch is much longer than all other scale lengths. In particular, space-charge effects are neglected during the interaction, which is assumed to take place immediately after the LWFA region. Within this context, the input to the code is a table listing the six-dimensional position and momentum coordinates of each macroparticle at a given time; from that data, the macroparticles are ballistically propagated until they are subjected to the incident laser pulse, and scattering occurs. The temporal information of the $\mathrm{x}$-ray pulse is calculated from the time of the interaction at each time step in conjunction with the time of flight of the produced photon to the detector at a specified distance to the interaction. Spatial information of the scattered $\mathrm{x}$-ray pulses can be determined by performing this calculation for several different observation angles.

Finally, we note that this code is linear; in other words, the square of the normalized vector potential, $A_{0}^{2}=$ $4 n_{\lambda} \lambda_{0} r_{0} \lambda_{C}$, is assumed to be much smaller than unity. More specifically, one must compare the different sources of spectral broadening of the x-ray spectrum: the relative bandwidth of the scattering laser, $\Delta \omega_{0} / \omega_{0}$; the relative energy spread of the electron beam, $2 \Delta \gamma / \gamma_{0}$; the momentum spread due to emittance, $\Delta u_{\perp} / u_{0}=\varepsilon / \gamma_{0} \sigma_{r}$; and the inhomogeneous radiation pressure, driven by $A_{0}^{2}$, as the Doppler-upshifted photon energy scales as

$$
\frac{k_{s}}{k_{0}} \simeq \frac{\gamma_{0}-u_{0} \cos \varphi}{\gamma_{0}-u_{0} \cos \theta} \frac{1}{1+\left\langle A_{0}^{2}\right\rangle},
$$

in the nonlinear regime [39]; one must check that $A_{0}^{2} \lessgtr$ $\max \left(2 \Delta \gamma / \gamma_{0}, \Delta \omega / \omega_{0}, \varepsilon / \gamma_{0} \sigma_{r}\right)$.

\section{X-RAY SIMULATION RESULTS}

As mentioned above, the input to the code is a table listing the six-dimensional position and momentum coordinates of each macroparticle at a given time; for the simulations given here, that input is directly provided by the PIC code describing the LWFA process. Figures 2 and 3 show the LWFA electron phase space, as simulated by the PIC code. As seen in Fig. 3, the phase space is highly correlated, most probably because of the strong nonlinear electromagnetic fields present in the laser-driven plasma wakefield. The electron bunch produced by LWFA is also extremely short; thus, 180-degree Compton scattering will provide equally short $\mathrm{x}$-ray flashes, of great potential interest to the users community; this is confirmed by the simulations, which show that a high $\mathrm{x}$-ray photon number can be produced: $2.95 \times 10^{7}$ photons, in the particular case simulated here, with a peak flux exceeding $4 \times$ $10^{21}$ photons/s. The main Compton scattering drive laser simulation parameters are summarized in Table II, where different sources of spectral broadening are also listed.

The x-ray spectrum shown in Fig. 4 is quite broad; this is due in part to the energy spread of the LWFA electron bunch, and to the relatively large divergence of the electron beam, which is also reflected in the x-ray beam, as shown in Fig. 5 (bottom); such broad spectra are ideal for ultrafast Laue diffraction experiments. The physical origin of the $\mathrm{x}-$ ray spectral broadening due to electron beam energy spread and divergence can be understood by considering Eq. (1), which describes the value of the scattered photon energy as a function of the electron energy, and the incidence and scattering angles. Fixing all other parameters,

TABLE II. X-ray code parameters.

\begin{tabular}{ll}
\hline \hline Drive laser pulse wavelength & $800 \mathrm{~nm}$ \\
Drive laser pulse energy & $50 \mathrm{~mJ}$ \\
Drive laser pulse $1 / e^{2}$ duration & $21 \mathrm{fs}$ \\
$1 / e^{2}$ focal radius & $40 \mu \mathrm{m}$ \\
Square of the normalized potential & $A_{0}^{2}=0.035$ \\
Electron beam relative energy spread & $2 \Delta \gamma / \gamma_{0} \simeq 0.1$ \\
Drive laser relative bandwidth & $\Delta \omega / \omega_{0} \simeq 0.06$ \\
Emittance broadening & $\varepsilon / \gamma_{0} \sigma_{r} \simeq 0.01$ \\
\hline \hline
\end{tabular}




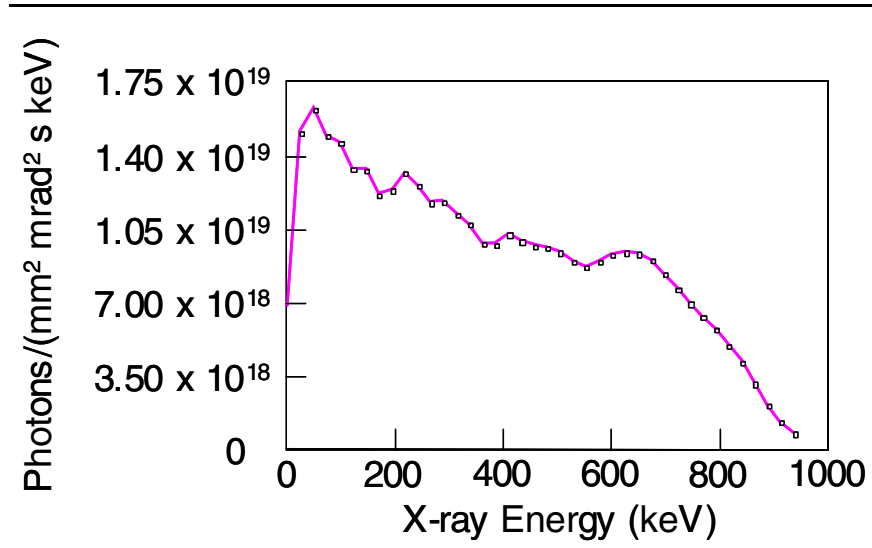

FIG. 4. (Color) On-axis X-ray spectrum, simulated by the threedimensional time and frequency domain Compton scattering code, using the three-dimensional PIC code-generated electron beam phase space as an input.

and taking into account the relation $\gamma=\sqrt{1+u^{2}}$, the scattered frequency is seen to vary quadratically with the electron energy; in terms of divergence, for those electrons propagating at a small angle with respect to the direction of observation, the Doppler upshift does not reach its maximum value, thus leading to low frequency components in the x-ray spectrum. We also note that the emittance correlates the electron bunch transverse size and its divergence: in this respect, one could envision manipulating the electron bunch phase space prior to the interaction to increase its size to match that of the Compton scattering laser (40 $\mu \mathrm{m} 1 / e^{2}$ focal radius), while lowering its divergence. This, however, would require fairly achromatic magnetic lenses which have not been designed for the present work. The electron bunch temporal shape is faithfully reproduced in the x-ray pulse, as seen in Fig. 5 (top), which opens the possibility of preparing specific electron beam pulse shapes for different x-ray applications; in particular, double pulsing the source on the femtosecond time scale appears feasible, in principle. At this point, it is important to reiterate that, in the present simulations, no effort was made to capture and manipulate the LWFA beam prior to $x$ ray production; depending upon the quantity of interest to the users, different configurations could be proposed: for example, relatively narrow-band $\mathrm{x}$-rays can be produced by increasing the electron beam size, while decreasing its divergence; on the other hand, for some other applications the $\mathrm{x}$-ray source size could be minimized, at the expense of spectral purity. Under the electron beam conditions simulated here, the peak $\mathrm{x}$-ray brightness exceeds $>10^{20}$ photons $/\left(\mathrm{mm}^{2} \mathrm{mrad}^{2} \mathrm{~s} 0.1 \%\right.$ bandwidth $)$, which represents a very high value for such a compact system; the average brightness remains modest, but multi-Joule, $\mathrm{kHz}$-class laser systems are currently under development, and could yield a path to improved average brightness, useful for advanced biomedical imaging and other advanced applications.
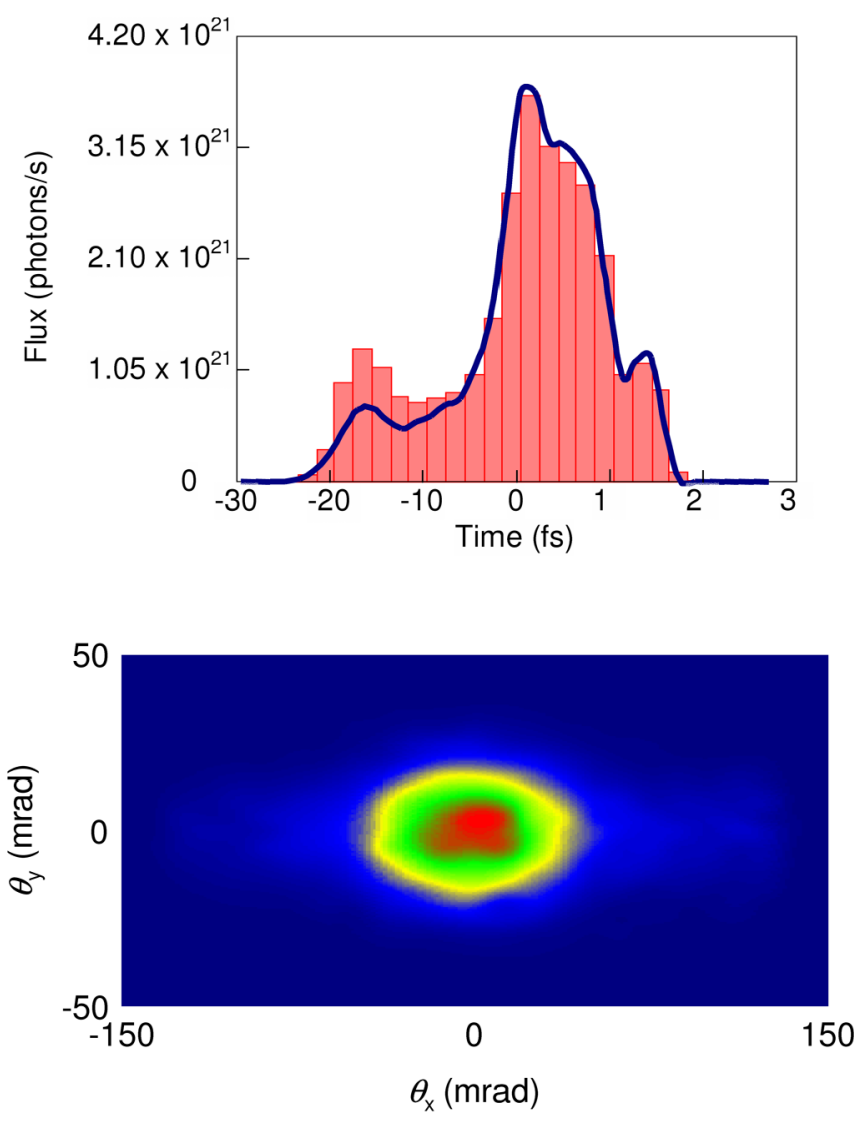

FIG. 5. (Color) Top: temporal x-ray pulse shape, as simulated by the three-dimensional time and frequency domain Compton code, using the three-dimensional PIC code-generated electron beam phase space as an input. The temporal x-ray pulse (blue line) is superimposed on the temporal electron distribution to show the close correlation. Bottom: x-ray angular energy distribution.

In closing, a brief comparison between LWFA-driven and linac-driven electron beams is given, within the context of Compton scattering. Key experimental parameters are listed in Table III, where the linac parameters represent the current state-of-the art for a room temperature S-band system; the energy of $300 \mathrm{MeV}$ corresponds to recently published LWFA results [35], and is of interest because it yields photon energies in the $\mathrm{MeV}$ range, where applications such as pair production on the femtosecond time scale

TABLE III. LWFA and linac parameters.

\begin{tabular}{lll}
\hline \hline & LWFA & S-band linac \\
\hline Energy & $300 \mathrm{MeV}$ & $300 \mathrm{MeV}$ \\
Gradient & $>10 \mathrm{GeV} / m$ & $25 \mathrm{MeV} / m$ \\
Energy spread & $<5 \%$ & $0.1 \%$ \\
Normalized emittance & $5 \mathrm{~mm} \mathrm{mrad}$ & $1 \mathrm{~mm} \mathrm{mrad}$ \\
Charge & $1 \mathrm{nC}$ & $1 \mathrm{nC}$ \\
Bunch duration & $10 \mathrm{fs}$ & $10 \mathrm{ps}$ \\
Laser-electron timing jitter & $\mathrm{fs}$ & $<\mathrm{ps}$ \\
\hline \hline
\end{tabular}


and nuclear resonance fluorescence become feasible. In terms of repetition rates, both systems scale up to $1 \mathrm{kHz}$.

Table III clearly shows the respective advantages and drawbacks of each technology: the LWFA is potentially a much more compact approach to bright electron beams, and produces extremely short bunches; linac-based systems still offer much lower energy spreads, at the expense of peak current and accelerating gradient. We also note that one can compress the electron bunches produced by a linac, but at the expense of energy spread, in a manner analogous to the correlation between electron beam spot size and divergence, measured in terms of emittance. As the electron beam phase space is the key influence on the $\mathrm{x}$-ray phase space, the respective advantages of each technology in terms of electron beam translate directly to the $\mathrm{x}$-ray parameter space.

\section{CONCLUSIONS}

Following the recent demonstration of efficient, high quality electron beam acceleration in a laser wakefield, three-dimensional simulations have been performed to generate an accurate picture of the electron beam phase space, and to predict the x-ray output of LWFA-based Compton scattering experiments, where the electron beam collides with a fraction of the drive laser pulse to produce a very bright, femtosecond x-ray flash. Our study, using fully benchmarked codes, shows that high x-ray photon number ( $>10^{7}$ photons/shot), high peak brightness $\quad\left[>10^{20}\right.$ photons $/\left(\mathrm{mm}^{2} \mathrm{mrad}^{2} \mathrm{~s} 0.1 \%\right.$ bandwidth $\left.)\right]$ $\mathrm{x}$-ray pulses could be produced following that approach. As the LWFA electron beam is extremely short, the maximum photon flux predicted by the code exceeds $10^{21}$ photons/s, and opens a path to ultrafast Laue diffraction experiments, taking advantage of the broadband $\mathrm{x}$-ray spectrum generated by Compton scattering. Finally, we note that the maximum x-ray energy produced in these simulations approaches $1 \mathrm{MeV}$, thus indicating that femtosecond positron pulses could also be generated by increasing either the LWFA electron beam energy or by frequency-multiplying the laser pulse used for Compton scattering to exceed the pair production threshold, and reach sufficiently large pair production cross section in dense metallic targets.

\section{ACKNOWLEDGMENTS}

This work was performed under the auspices of the U.S. Department of Energy by University of California, Lawrence Livermore National Laboratory, under Contract No. W-7405-Eng-48. One of us (F. V.H.), would also like to acknowledge very useful discussions with $\mathrm{D}$. T. Santa Maria.

[1] N. Bloembergen, Rev. Mod. Phys. 71, S283 (1999).
[2] A. Rousse et al., Rev. Mod. Phys. 73, 17 (2001).

[3] http://tesla.desy.de/new_pages/TDR_CD/PartV/fel.html

[4] http://www-ssrl.slac.stanford.edu/lcls/

[5] J. Drenth, Principles of Protein X-Ray Crystallography (Springer, New York, 1999), 2nd ed.

[6] A. Rousse et al., Phys. Rev. E 50, 2200 (1994).

[7] Ch. Rischel et al., Nature (London) 390, 490 (1997).

[8] C. W. Siders, A. Cavalleri, K. Sokolowski-Tinten, Cs. Toth, T. Guo, M. Kammler, M. Horn von Hoegen, K. R. Wilson, D. von der Linde, and C. P. J. Barty, Science 286, 1340 (1999).

[9] R. Bartels, S. Backus, E. Zeek, L. Misoguti, G. Vdovin, I. P. Christov, M. M. Murnane, and H. C. Kapteyn, Nature (London) 406, 164 (2000).

[10] J. Arthur, G. Materlik, R. Tatchyn, and H. Winick, Rev. Sci. Instrum. 66, 1987 (1995).

[11] D. J. Gibson, S. G. Anderson, C. P. J. Barty, S. M. Betts, R. Booth, W. J. Brown, J. K. Crane, R. R. Cross, D. N. Fittinghoff, F. V. Hartemann, J. Kuba, G. P. Le Sage, D. R. Slaughter, A. M. Tremaine, A. J. Wootton, E. P. Hartouni, P. T. Springer, and J. B. Rosenzweig, Phys. Plasmas 11, 2857 (2004).

[12] A. Ting et al., Nucl. Instrum. Methods Phys. Res., Sect. A 375, ABS68 (1996).

[13] I. V. Pogorelsky et al., Phys. Rev. ST Accel. Beams 3, 090702 (2000).

[14] W. P. Leemans et al., Phys. Rev. Lett. 77, 4182 (1996).

[15] K. Chouffani et al., Nucl. Instrum. Methods Phys. Res., Sect. A 495, 95 (2002).

[16] Y. Li et al., Phys. Rev. ST Accel. Beams 5, 044701 (2002).

[17] J. Faure, Y. Glinec, A. Pukhov, S. Kiselev, S. Gordienko, E. Lefebvre, J.-P. Rousseau, F. Burgy, and V. Malka, Nature (London) 431, 541 (2004).

[18] S.P. D. Mangles, C.D. Murphy, Z. Najmudin, A. G. R. Thomas, J. L. Collier, A.E. Dangor, E. J. Divall, P. S. Foster, J. G. Gallacher, C. J. Hooker, D. A. Jaroszynski, A. J. Langley, W. B. Mori, P. A. Norreys, F. S. Tsung, R. Viskup, B.R. Walton, and K. Krushelnick, Nature (London) 431, 535 (2004).

[19] C. G. R. Geddes, Cs. Toth, J. van Tilborg, E. Esarey, C. B. Schroeder, D. Bruhwiler, C. Nieter, J. Cary, and W. P. Leemans, Nature (London) 431, 538 (2004).

[20] P. Catravas, E. Esarey, and W. P. Leemans, Meas. Sci. Technol. 12, 1828 (2001).

[21] A. Ting, C. I. Moore, K. Krushelnick, C. Manka, E. Esarey, P. Sprangle, R. Hubbard, H. R. Burris, R. Fischer, and M. Baine, Phys. Plasmas 4, 1889 (1997).

[22] F. Amiranoff, S. Baton, D. Bernard, B. Cros, D. Descamps, F. Dorchies, F. Jacquet, V. Malka, J. R. Marqès, G. Matthieussent, P. Miné, A. Modena, P. Mora, J. Morillo, and Z. Najmudin, Phys. Rev. Lett. 81, 995 (1998).

[23] C. Gahn, G. D. Tsakiris, A. Pukhov, J. Meyer-ter-Vehn, G. Pretzler, P. Thirlof, D. Habs, and K. J. Witte, Phys. Rev. Lett. 83, 4772 (1999);

[24] R. F. Hubbard, P. Sprangle, and B. Hafizi, IEEE Trans. Plasma Sci. 28, 1159 (2000).

[25] D. Umstadter, Phys. Plasmas 8, 1774 (2001).

[26] A. Pukhov, S. Gordienko, S. Kiselev, and I. Kostyukov, Plasma Phys. Controlled Fusion 46, B179 (2004). 
[27] A. Pukhov and S. Gordienko, Phil. Trans. R. Soc. A 364, 623 (2006).

[28] H. Schwoerer, B. Liesfield, H.-P. Schlenvoigt, K.-U. Amthor, and R. Sauerbrey, Phys. Rev. Lett. 96, 014802 (2006).

[29] A. Pukhov and J. Meyer ter Vehn, Appl. Phys. B 74, 355 (2002).

[30] I. Kostyukov, A. Pukhov, and S. Kiselev, Phys. Plasmas 10, 4818 (2003).

[31] F. V. Hartemann, W. J. Brown, D. J. Gibson, S. G. Anderson, A. M. Tremaine, P. T. Springer, A. J. Wootton, E. P. Hartouni, and C. P. J. Barty, Phys. Rev. ST Accel. Beams 8, 100702 (2005).

[32] W. J. Brown and F. V. Hartemann, Phys. Rev. ST Accel. Beams 7, 060703 (2004).

[33] W. J. Brown, S. G. Anderson, C. P. J. Barty, S. M. Betts, R. Booth, J. K. Crane, R. R. Cross, D. N. Fittinghoff, D. J. Gibson, F. V. Hartemann, E. P. Hartouni, J. Kuba, G. P. Le Sage, J. B. Rosenzweig, D. R. Slaughter, A. M. Tremaine, A. J. Wootton, and P. T. Springer, Phys. Rev. ST Accel.
Beams 7, 060702 (2004).

[34] F. V. Hartemann, A. M. Tremaine, S. G. Anderson, C. P. J. Barty, S. M. Betts, R. Booth, W. J. Brown, J. K. Crane, R. R. Cross, D. J. Gibson, D. N. Fittinghoff, J. Kuba, G. P. Le Sage, D. R. Slaughter, A. J. Wootton, E. P. Hartouni, P. T. Springer, J. B. Rosenzweig, and A. K. Kerman, Laser Part. Beams 22, 221 (2004).

[35] J. Faure, C. Rechatin, A. Norlin, A. Lifschitz, Y. Glinec, and V. Malka, Nature (London) 444, 737 (2006).

[36] E. Esarey, R. F. Hubbard, W. P. Leemans, A. Ting, and P. Sprangle, Phys. Rev. Lett. 79, 2682 (1997).

[37] S. Fritzler, E. Lefebvre, V. Malka, F. Burgy, A. E. Dangor, K. Krushelnick, S.P.D. Mangles, Z. Najmudin, J.-P. Rousseau, and B. Walton, Phys. Rev. Lett. 92, 165006 (2004).

[38] L. M. Brown and R. P. Feynman, Phys. Rev. 85, 231 (1952).

[39] F. V. Hartemann, High-Field Electrodynamics (CRC Press, Boca Raton, FL, 2002). 